\title{
A Generic Polycarbonate Based Microfluidic Tool to Study Crystal Nucleation in Microdroplets
}

\author{
Daniel Selzer, Burkard Spiegel, Matthias Kind* \\ Institute of Thermal Process Engineering, Karlsruhe Institute of Technology (KIT), Karlsruhe, Germany \\ Email: *matthias.kind@kit.edu
}

How to cite this paper: Selzer, D., Spiegel, B. and Kind, M. (2018) A Generic Polycarbonate Based Microfluidic Tool to Study Crystal Nucleation in Microdroplets. Journal of Crystallization Process and Technology, 8, 1-17.

https://doi.org/10.4236/jcpt.2018.81001

Received: October 18, 2017

Accepted: November 30, 2017

Published: December 4, 2017

Copyright ( 92018 by authors and Scientific Research Publishing Inc. This work is licensed under the Creative Commons Attribution International License (CC BY 4.0).

http://creativecommons.org/licenses/by/4.0/ (c) (i) Open Access

\begin{abstract}
Crystal nucleation is important to control the product properties in industrial crystallization processes. To investigate crystallization phenomena, methods which rely on microscopic volumes have gained relevance over the last decade. Microfluidic devices are suitable for carrying out crystallization experiments based on a large set of individual droplets in the nanoliter range. In this work, we propose a simple method to manufacture such devices from polycarbonate as an alternative to conventional chips made of poly (dimethylsiloxane). The microfluidic device consists of two main functional parts: A $\mathrm{T}$-junction for droplet generation and a section for storage and observation of up to 400 individual droplets. Using these manufactured devices, it is easy to produce and store highly monodisperse droplets of substances that require either a hydrophilic or hydrophobic surface of the microchannel. Since crystal nucleation is a stochastic process which depends on the sample volume, a reproducible droplet volume is of great importance for crystallization experiments. The versatile applicability of the manufactured devices is demonstrated for substances which are used in different crystallization applications, for example, solution crystallization (aqueous potassium nitrate solution) and melt crystallization (ethylene glycol distearate). Finally, we demonstrate that the manufactured microfluidic devices in our experimental setup can be used to conduct crystal nucleation measurements. Based on these measurements we discuss our results with respect to state-of-the-art nucleation models.
\end{abstract}

\section{Keywords}

Microfluidics, Polycarbonate, Crystallization, Electrolyte Solution, Organic Melt 


\section{Introduction}

Crystallization in small sample volumes is important for a variety of industrial crystallization processes (e.g. emulsion crystallization processes [1] [2], flashcrystallization [3]). A powerful method to investigate crystallization phenomena in discrete volumes is microfluidics [4]. Microfluidics, for example, has been widely used in the field of protein crystallization [5]-[12]. Furthermore, this technique has been used as a tool to perform crystallization experiments in the field of solution crystallization [9] [13] [14] [15] [16]. Since crystal nucleation in small volumes is a stochastic process, experiments have to be carried out on a large set of independent "microreactors" [17] [18] [19]. Using the classical droplet method, these microreactors are represented by independent microdroplets. A reproducible droplet volume and a high level of monodispersity of the micro droplets is an essential condition for kinetic measurements, as crystal nucleation can be influenced by droplet volume. The continuous phase needs to preferentially wet the channel walls throughout the dispersed phase so that the microfluidic device can form droplets. Partial wetting of the channel walls in the dispersed phase would lead to instable droplet generation [20]. This can be overcome by adjusting the wetting properties of the channel walls either hydrophobically or hydrophilically. Under these conditions, contact between the dispersed phase and the channel walls is prevented due to a thin layer of the continuous phase [21] [22]. Since the channel walls may act as active nucleation centers, this is also of great importance regarding crystal nucleation experiments.

The choice of the material is of crucial importance in the fabrication of microfluidic devices, since the properties of the material influences both the fabrication process and the application of the device. A wide range of materials, such as glass, steel, poly (methylmetacrylate) (PMMA), polycarbonate (PC), poly (dimethylsiloxane) (PDMS), polytetrafluorethylene (PTFE) or polyolefine have been used for the fabrication of microfluidic chips [23] [24]. A key material in the production of microfluidic devices is PDMS. The main advantages of this material are, for example, low cost, optical transparency in the UV-visible regions, chemical inertness and flexibility [25] [26].

However, PDMS also has some disadvantages which limit its application to aqueous reactions due to its very low resistance to organic solvents [27]. One major drawback is that PDMS swells when in contact with certain substances (e.g. hexadecane, silicone oil and toluene) due to its porous nature. This can lead to a significant deformation of the channel geometry [28] [29]. Consequently, hydrodynamic flow conditions may change with increasing contact time between the solvent and the microfluidic chip. Thus, reproducible droplet generation and droplet volume can be affected.

An alternative material is PC, which has already been used for microfluidic experiments by some research groups [12] [30] [31]. The benefits of this material lie in its low moisture absorption, good machining properties, transparency in the visible range, biocompatibility and high glass transition tempera- 
ture $\left(T_{\mathrm{g}} \approx 148^{\circ} \mathrm{C}\right)$ [20] [32] [33]. Furthermore, swelling problems, which are known to occur with PDMS devices when silicone oil is used [29], can be avoided with microfluidic devices made of PC. Since silicone oil offers some great advantages as a carrier fluid for the generation and storage of microdroplets containing an aqueous phase (e.g. chemical inertness, low cost compared to fluorocarbon oils, a wide variety of different viscosities are available), PC is particularly interesting for crystal nucleation measurements of aqueous solutions and water droplets. Polycarbonate also enables microfluidic crystallization experiments on organic melts (e.g. hexadecane) [34] without deformation of the microfluidic geometry due to swelling.

To the best of our knowledge, the advantages of PC mentioned above have not been used for the investigation of crystal nucleation. In this work, we use a simple method to produce microfluidic devices from PC as an alternative to conventional chips made of PDMS. These devices can produce and store highly monodisperse droplets for substances that require hydrophilic or hydrophobic surface wetting properties without the need for sophisticated apparatuses. As the capability of reproducible droplet formation is of great importance for crystallization experiments on a large set of individual microdroplets, we also show that our microfluidic setup is suitable for stable droplet formation. Furthermore, we demonstrate that the microfluidic devices produced by this method can be used to conduct crystal nucleation measurements under well-defined conditions. The versatile applicability of the manufactured devices is demonstrated for substances which are used in different crystallization applications, for example, solution crystallization (aqueous potassium nitrate solution) and melt crystallization (ethylene glycol distearate). Finally, we discuss different nucleation models applied to our experimental data.

\section{Materials and Methods}

\subsection{Materials and Reagents}

\section{Fabrication of chips}

Microfluidic channels were fabricated in PC slabs (Makrolon ${ }^{\circledR}$, Bayer) with a thickness of $2 \mathrm{~mm}$ by micromilling. After milling, we sealed the PC blanks by bonding the devices with a PC foil (Makrolon ${ }^{\circledR}$, Bayer) with a thickness of 250 $\mu \mathrm{m}$.

\section{Hydrophilic and hydrophobic surface modification}

We prepared two different solutions for the hydrophilic and hydrophobic surface modification of the PC. Hydrophilic modification was performed by using a solution of Tin (II) chloride dihydrate ( $\geq 98 \%$, Carl Roth, Germany) in ethanol ( $\geq 99.9 \%$, Merck, Germany). Hydrophobic surface modification was performed by preparing a solution of dodecylamine (DDA, $\geq 99 \%$, Sigma-Aldrich, Germany) in ethanol. All chemicals were used without further purification.

\section{Crystallization experiments}

We used ethylene glycol distearate (EGDS, melting temperature $T_{\text {melt }}=$ 
$60.5^{\circ} \mathrm{C}$ ) with a mass purity of $\geq 98 \%$ (Wako Chemicals, Germany) and a solution of potassium nitrate $\left(\mathrm{KNO}_{3}\right)$ with a mass purity of $\geq 99 \%$ (Carl Roth, Germany) in deionized water $(18.2 \mathrm{M} \Omega \cdot \mathrm{cm}$, Millipore) for the crystallization experiments. The concentration of the $\mathrm{KNO}_{3}$ solution was set to $x_{\mathrm{s}}=0.367 \mathrm{~kg}_{\mathrm{KNO} 3} / \mathrm{kg}_{\text {sol }}\left(T_{\text {eq }}=\right.$ $35^{\circ} \mathrm{C}$ ) for all experiments. Deionized water was used as a continuous phase to generate droplets of EGDS, whereas droplets of $\mathrm{KNO}_{3}$ solution were generated in viscous silicon oil (350 mPas, Carl Roth, Germany). The $\mathrm{KNO}_{3}$ solution was filtered by means of a syringe filter $(0.2 \mu \mathrm{m})$ prior to the experiments. All chemicals were used without further purification.

\subsection{Fabrication of Polycarbonate Chip}

We designed the geometry of the devices by means of computer-aided design (CAD) software (PTC Creo Parametric) before milling the channels of the microfluidic device in the PC slab. A milling machine (WF4 CNC, Kunzmann, Germany) equipped with a high-performance milling spindle (Type: 832-022, Henninger, Germany) was used for the milling process. Depending on the chip design, the milling process took around $2 \mathrm{~h}$. With this setup, we can manufacture the channels in the PC slabs accurately by using two flute micro end-mills (Kempf, Germany) with a diameter of $200 \mu \mathrm{m}$ and $300 \mu \mathrm{m}$, respectively, at a maximum revolution speed of $30,000 \mathrm{rpm}$. The mills were cooled by an oil-in-water emulsion during the entire milling process. The micro end-mills used were replaced every second microfluidic chip to achieve constant channel properties. We measured constant deviations of approximately $5 \%-10 \%$ of the nominal channel geometry. The chip blanks were cleaned from debris with pressurized air and rinsed with detergent and deionized water after milling. The chip blanks were then sealed with PC foil with a thickness of $250 \mu \mathrm{m}$ via thermal bonding close to the glass transition temperature of PC $\left(T_{\mathrm{g}} \approx 148^{\circ} \mathrm{C}\right)$ at $T_{\mathrm{b}}=$ $146^{\circ} \mathrm{C}$ for $2 \mathrm{~h}$ in a circulating air oven (Memmert, Germany). During this process, the chip blanks and the foil were pressed together between two glass plates under constant pressure by using four brackets. The sealed chip was removed from the oven as soon as the latter had cooled to approximately $80^{\circ} \mathrm{C}$. Finally, commonly available $800 \mu \mathrm{m}$ metal cannulas (Braun, Germany) equipped with silicon sleeves were used as in- and outlets of the microfluidic device.

\subsection{Surface Modification and Characterization}

Hydrophobic and hydrophilic surface modification of the microfluidic device was carried out by methods described elsewhere in detail [20] [32]. Hydrophobic surface modification was achieved by flowing a solution of DDA (5 wt\%) in ethanol through the channels at a constant flow rate $(1000 \mu \mathrm{l} / \mathrm{h})$ and temperature $\left(60^{\circ} \mathrm{C}\right)$ for $2 \mathrm{~h}$. A solution of $\mathrm{SnCl}_{2}(20 \mathrm{wt} \%)$ in ethanol was pumped through the microfluidic device at a flow rate of $400 \mu \mathrm{l} / \mathrm{h}$ at otherwise identical conditions for hydrophilic modification. The wettability of unmodified hydrophilic and hydrophobic PC slabs was measured by using a sessile drop method on a contact 
angle system (G10, Krüss, Germany) at ambient temperature. The PC samples were cleaned with isopropyl alcohol and deionized water. Immediately after cleaning, deionized water was placed on the surface of the samples by using a syringe and its static contact angles were measured at least three times. The topographic property of the microchannel surface was characterized by scanning electron microscopy (Leo Evo 50, Carl Zeiss, Germany) and measured by confocal white light microscope (NANOFOCUS $\mu$ SURF, Nanofocus, Germany). The arithmetic average of the arithmetical mean roughness value $R_{\mathrm{a}}$ and the total height of the roughness profile $R_{\mathrm{t}}$ was computed after filtering and separating high- and low-frequency components to specify the surface roughness.

\subsection{Experimental Setup and Chip Design}

The device is integrated into the experimental microfluidic setup to perform crystallization experiments. The setup consists of a CCD camera (pco.edge 5.5, PCO AG, Germany) coupled to a stereo microscope (SZ61, Olympus), a box with two syringe pumps (CETONI, Germany) thermostatted by a heating fan (LE Mini Sensor, Leister Technologies, Germany) and a temperature control unit (see Figure 1(b)) for the microfluidic device, as illustrated in Figure 1(a). Supersaturation can be built up quickly by cooling the microfluidic device using a Peltier element (QUICK-OHM Küpper, Germany) to induce crystallization. An aluminum slab with a thickness of $2 \mathrm{~mm}$ covered with a heat conductive foil (KERAFOL, Germany) is placed between the Peltier element and the microfluidic device to avoid small temperature gradients. Since the exhaust heat of the Peltier element needs to be removed, the Peltier element is placed on a thermostatted aluminum block. During crystallization experiments, this block is kept at a
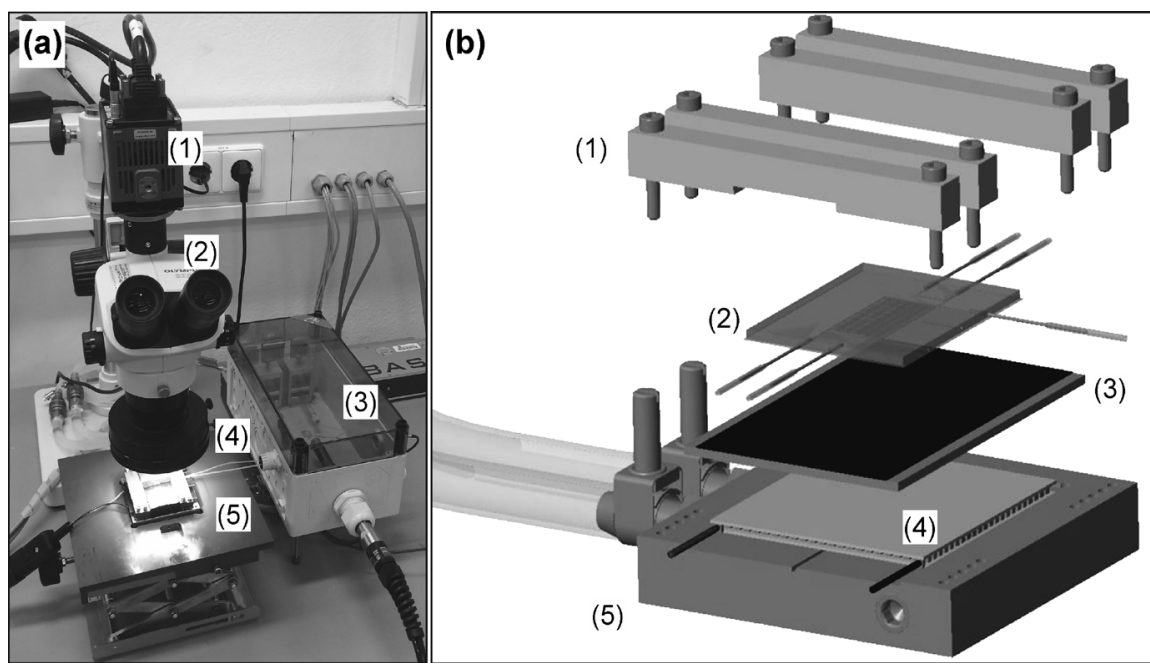

Figure 1. (a) Experimental microfluidic setup: (1) CCD camera, (2) microscope, (3) thermostatted box with two syringe pumps, (4) polarizer and (5) measuring cell with microfluidic device. (b) 3D CAD model of the temperature control unit with microfluidic device: (1) chip mounting, (2) microfluidic device, (3) aluminum slab ( $s=2 \mathrm{~mm}$ ) with thermal conductive foil ( $s=0.2 \mathrm{~mm}$ ), (4) Peltier element and (5) thermostatted aluminum block. 

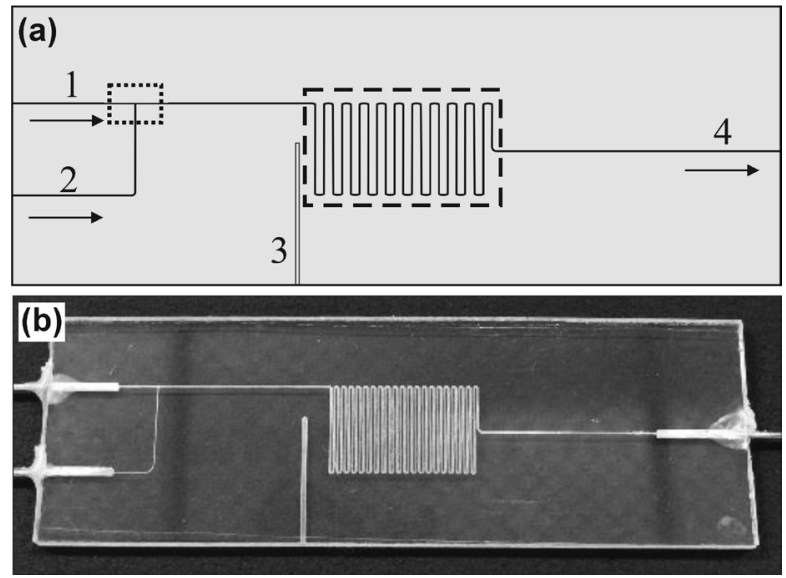

Figure 2. (a) Chip design and schematic illustration of the microfluidic device: inlets for the dispersed and continuous phase ((1), (2)), channel to insert a thermocouple (3), one outlet (4), the T-junction for droplet generation (marked by dotted line) and the storage area (marked by dashed line); (b) image of a fabricated microfluidic chip.

constant temperature by using a thermostat (LAUDA, Germany).

A schematic illustration and a photograph of a fabricated microfluidic chip are shown in Figure 2(a) and Figure 2(b), respectively. The microfluidic device is comprised of two inlets and one outlet. The dispersed phase is injected through inlet 1, whereas the continuous phase is injected via inlet 2 (see Figure $2(a)$ ) by two individual syringe pumps. Droplets are continuously generated via the T-junction (marked by the dotted line in Figure 2(a)) in the first part of the microfluidic device. The second part of the chip design consists of the storage area (marked by the dashed line in Figure 2(a)). The storage area consists of a serpentine shaped storage channel to observe as many droplets as possible during each crystallization experiment. Based on this chip design, up to 400 droplets can be stored during each crystallization experiment. The nominal channel dimensions were $300 \times 200 \mu \mathrm{m}$ for experiments with the aqueous solution of $\mathrm{KNO}_{3}$ and $200 \times 200 \mu \mathrm{m}$ for experiments with EGDS.

The precise knowledge of the temperature close to the storage channels is of great importance for crystallization experiments based on cooling crystallization. Therefore, thin thermocouples ( $d=500 \mu \mathrm{m}$, Electronic Sensor, Germany) are situated in and below the microfluidic device. Consequently, the actual temperature in the storage area can be estimated by assuming stationary heat transfer.

\section{Results and Discussion}

Our microfluidic device meets several requirements for crystal nucleation experiments based on cooling crystallization: i) Well-defined chip geometries and a smooth surface of the microchannel after fabrication to perform crystallization experiments as specified; ii) a stable surface modification to enable the production of oil-in-water or water-in-oil droplets and to prohibit interactions between the droplets and the channel walls that can otherwise influence crystallization; iii) production of almost monodisperse droplets with small derivations at dif- 
ferent sizes to study the volume effect on crystallization; and iv) precise experimental conditions (e.g. temperature). We demonstrate the suitability of our microfluidic device for these tasks in the following sections.

\subsection{Characterization of the Microfluidic Device}

Thermal bonding was applied to seal the microfluidic device with a cover foil after milling. This easy-to-use method can cause strong shape distortion of the channel geometry in comparison to chemical or adhesive bonding methods [24]. Therefore, we checked the shape of the channels by imaging clean-cut cross sections. Figure 3 shows the cross section of the chip before and after bonding. No significant shape dislocation is apparent. Obviously, the rectangular cross section is preserved during the bonding process. The positive connection between the cover foil and the PC slab is apparent in Figure 3(b) and Figure 3(d). It can be shown that no separation occurs during the experiments, even after weeks.

In addition to the macroscopic shape of the microchannels, the topographic quality is important for crystallization experiments. It is known that crystallization is sensitive to foreign particles or surfaces. A rough channel surface may lead to an undesired adherence of crystals or droplet deformation at emerged spots and, thus, impact crystallization. We quantified the surface roughness of the fabricated microfluidic device by confocal white light microscopy. The result is depicted exemplarily for a hydrophobically modified microchannel as a surface roughness plot in Figure 4(a). Figure 4(b) compares the $R_{\mathrm{a}}$ value for all modifications. Unmodified PC has a value of around $0.1 \mu \mathrm{m}$. Hydrophobic surface modification appears to lower this value, whereas an increased $R_{\mathrm{a}}$ value of $0.6 \mu \mathrm{m}$ is found for hydrophilic PC. The same trend is apparent for the maximum height of the profile $R_{\mathrm{t}}$. Hydrophilic modification causes the highest value of around $1.4 \mu \mathrm{m}$. Compared to common materials for crystallization vessels, for example, stainless steel STS316L with $R_{\mathrm{a}}$ in the range of $2.0 \mu \mathrm{m}$ (unpolished) to
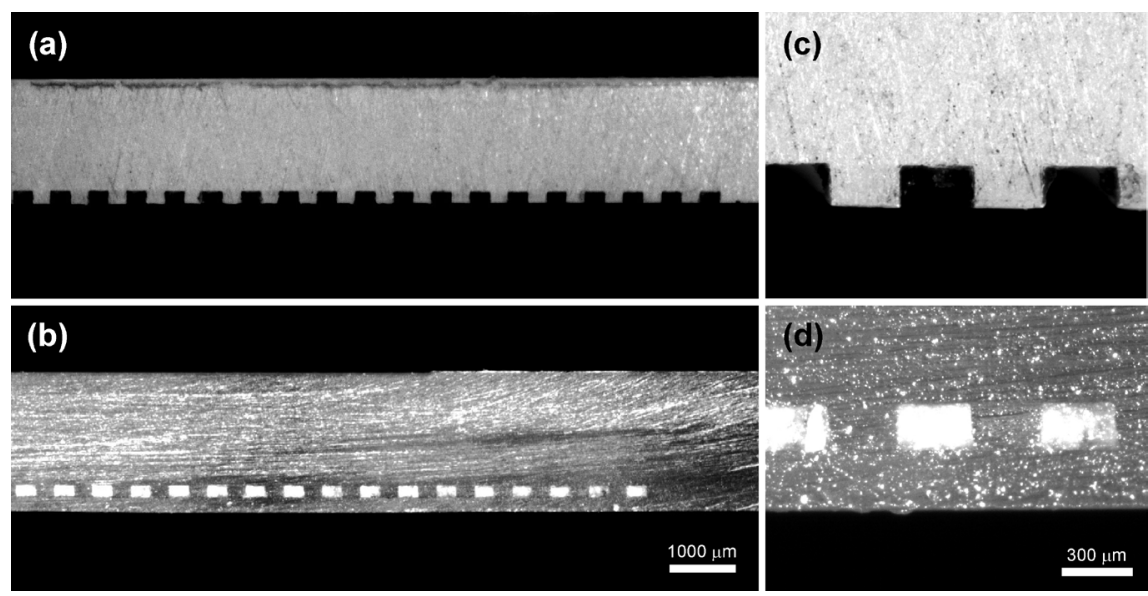

Figure 3. Images of clean-cut cross sections of the micro-channels before bonding ((a), (c)) and after bonding with a foil of PC $(250 \mu \mathrm{m})$ via thermal bonding at $T_{\mathrm{b}}=146^{\circ} \mathrm{C}((\mathrm{b})$,

(d)) in large-scale (left) and in detail (right). The white spots (debris) within the microchannels in picture (c) and (d) are due to the polishing procedure. 
(a)

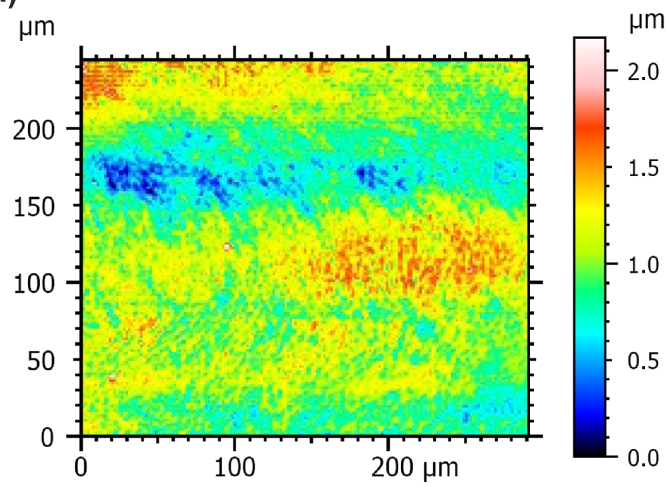

(b)

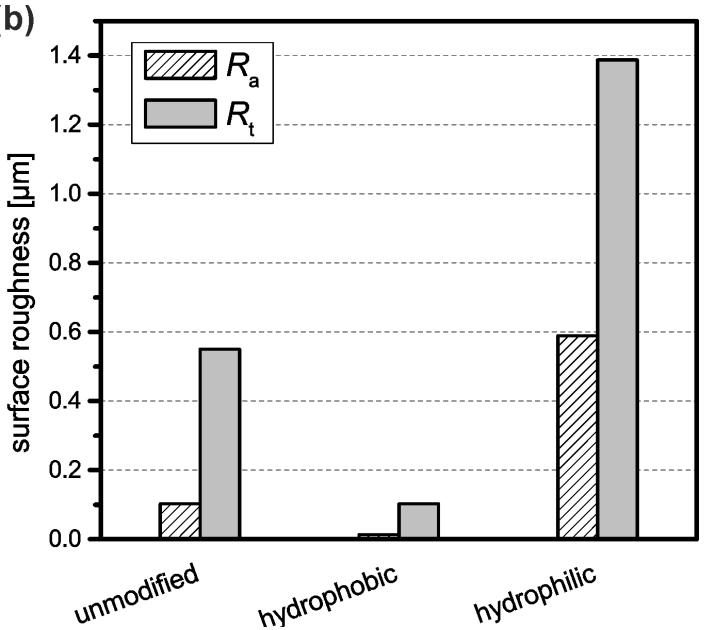

Figure 4. (a) Example of a typical surface roughness plot of a hydrophobically modified microchannel. The plot shows a section of the bottom of the microchannel; (b) arithmetical mean roughness value $R_{\mathrm{a}}$ (solid bar) and the value of the maximum height of the roughness profile $R_{\mathrm{t}}$ (shaded bar) of unmodified, hydrophobic and hydrophilic PC microchannels.

$0.1 \mu \mathrm{m}$ (mechanical polishing), the surface roughness $R_{\mathrm{a}}$ measured is acceptable [35].

The results obtained are supported by SEM micrographs (see Figure 5). Small irregular elevations at the surface of untreated PC, probably caused by the milling process, were visible. A different surface structure was found after hydrophobic modification (see Figure 5(b)). A high number of small ruptures occurred in a characteristic pattern, probably aligned in the direction of flow. Observations of [20] showed a different, more irregular surface structure. In contrast to our work, the surface of modified PC slabs was examined instead of milled microchannels. Changed surface properties due to the milling process and different flow conditions during surface modification could be a reasonable explanation. A flaky structure with extended cracks was found for hydrophilic PC. Comparing the images, a greater roughness of hydrophilic microchannels seems reasonable.

The most crucial aspect of surface modification is the adjustment of the wettability and, thus, the qualification for stable droplet production and storage. Both, the generation and storage of stable oil-in-water and water-in-oil droplets is not possible with untreated PC [20]. Partial wetting of the channels walls or separation of the two phases occurs. The advantage of PC as a chip material is the flexibility to shift the wettability in both directions by established and easy-to-use methods. We checked the successful application of these methods on PC slabs. The determined contact angles of water droplets are shown in Figure 6. The contact angle changes from $84^{\circ}$ for untreated PC to $125^{\circ}$ after hydrophobic modification. Consequently, the continuous silicone oil phase wets preferentially the channel walls and aqueous droplets will form (see Figure 7(a)). After hydrophilic modification the contact angle is $46^{\circ}$. Thus, the oil acts as dispersed phase and water as continuous phase (see Figure $7(b)$ ). Furthermore, we 

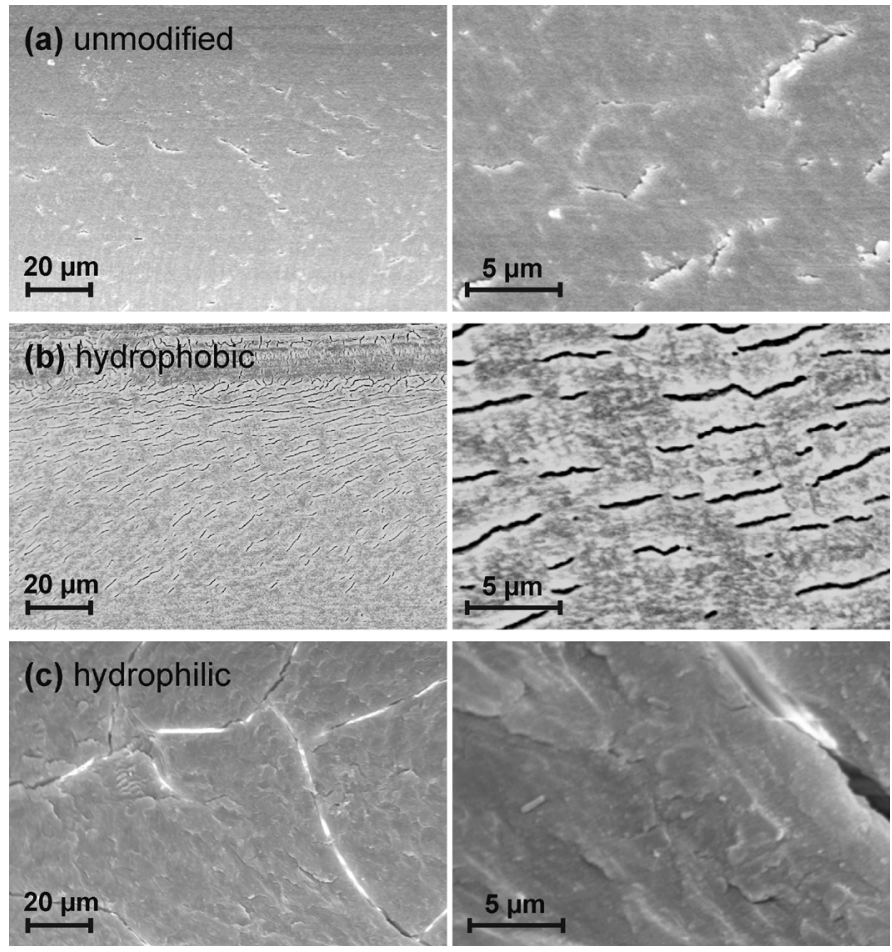

Figure 5. (a) SEM micrographs of the milled microchannel surface structures for unmodified, (b) hydrophobically and (c) hydrophilically modified PC in large-scale (left) and in detail (right).

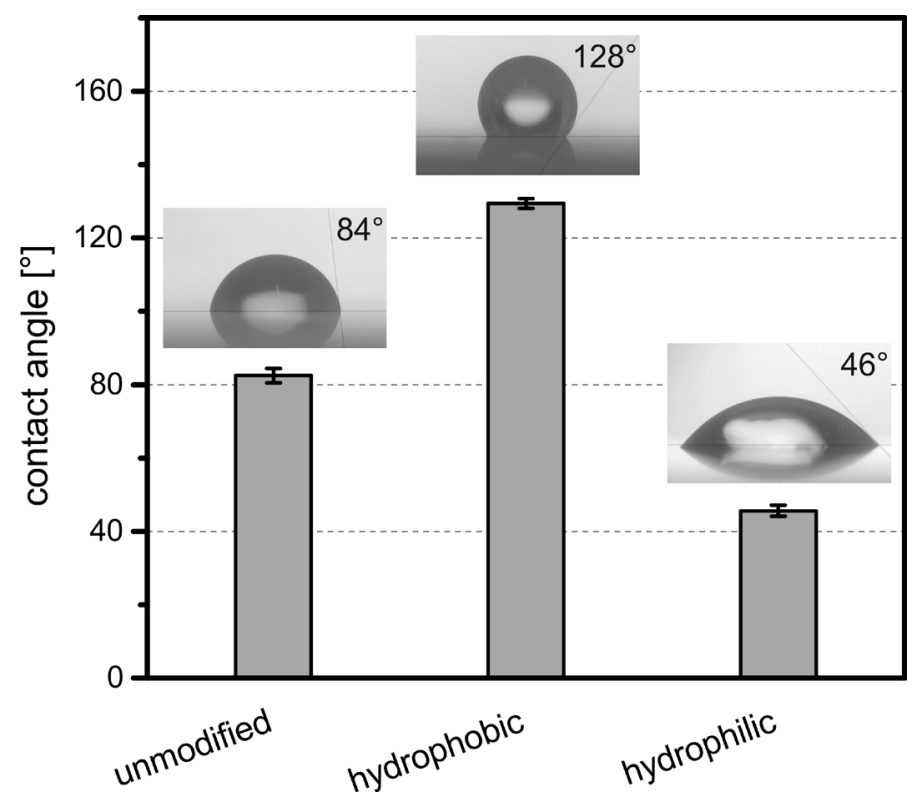

Figure 6. Contact angle of deionized water on different modified PC slabs by the sessile drop method and corresponding photographs of the droplets.

proof the successful adjustment of the surface wettability in the next chapter by a droplet-generation study. The microfluidic devices could produce stable droplets over a period of several weeks during the experiments, even at elevated temperatures up to $70^{\circ} \mathrm{C}$. 

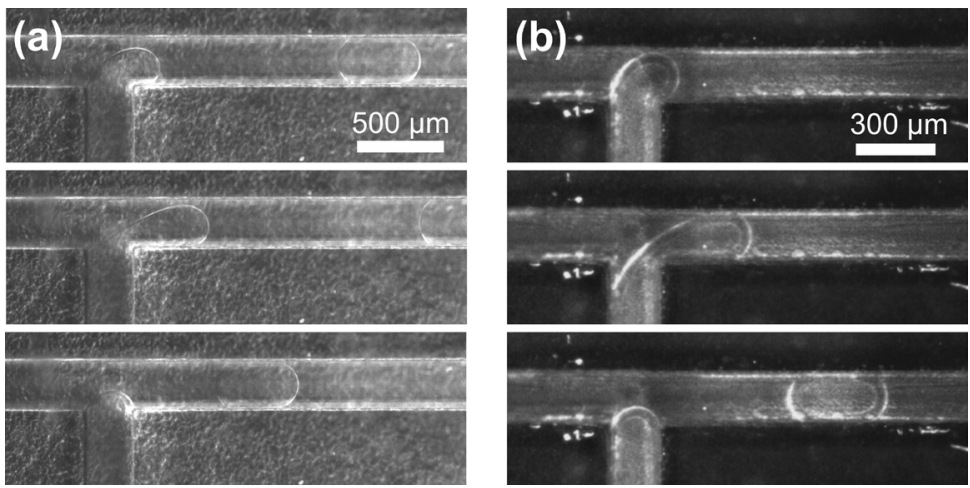

Figure 7. Generation of (a) $\mathrm{KNO}_{3}$ solution droplets in silicone oil (flow rate of the continuous phase $Q_{\mathrm{c}}=300 \mu \mathrm{l} / \mathrm{h}$ ) at a flow rate of the dispersed phase $Q_{\mathrm{d}}=120 \mu \mathrm{l} / \mathrm{h}$ and (b) EGDS droplets in water $\left(Q_{\mathrm{c}}=400 \mu \mathrm{l} / \mathrm{h}\right)$ at $Q_{\mathrm{d}}=80 \mu \mathrm{l} / \mathrm{h}$ via the T-junction of the microfluidic device.

\subsection{Droplet Generation}

Precise knowledge of the droplet volume is a crucial requirement in microfluidic experiments to determine crystal nucleation kinetics based on the classical droplet method. As crystal nucleation kinetics derived by means of the classical droplet method rely on a statistical analysis, a large set of droplets (microreactors) with a high degree of monodispersity is required.

In our work, droplets were generated via a $\mathrm{T}$-junction by injecting the dispersed and continuous phase through the inlets of the microfluidic device (see Figure 7). Flow rates were accurately (less than 1\%) controlled by two programmable syringe pumps (CETONI, Germany). The chip was kept above the liquidus temperature of the aqueous $\mathrm{KNO}_{3}$ solution $\left(T_{\text {eq }}=35^{\circ} \mathrm{C}\right)$ at $40^{\circ} \mathrm{C}$ and above the melting temperature of EGDS $\left(T_{\text {melt }}=60.5^{\circ} \mathrm{C}\right)$ at $70^{\circ} \mathrm{C}$ during the droplet generation process to avoid clogging of the channels.

It is possible to count the number of droplets $N_{\mathrm{d}}$ produced in a certain time $\tau$ elapsed between the first and last droplet in a recorded video sequence by recording a video sequence of the droplet generation process with a CCD camera. The mass of the dispersed phase injected by the syringe pump equals the sum of the mass of the droplets generated. Following this, the average droplet volume can be easily calculated as

$$
\bar{V}_{d}=\frac{Q_{d} \cdot \tau}{N_{d}}
$$

One important benefit of this method is that knowledge of the droplet and channel dimensions is not required. Since the droplets are surrounded by a thin film of the continuous phase, the exact droplet dimensions are hard to determine by optical methods. In addition, since some solvents induce swelling of the channels, the channel dimensions may change with time [28] [29]. Another drawback is that most microfluidic devices are made of flexible material, for example, plastic, which tends to expand as a response to pressure induced by the two-phase flow. This makes it difficult to calculate the droplet volume directly 

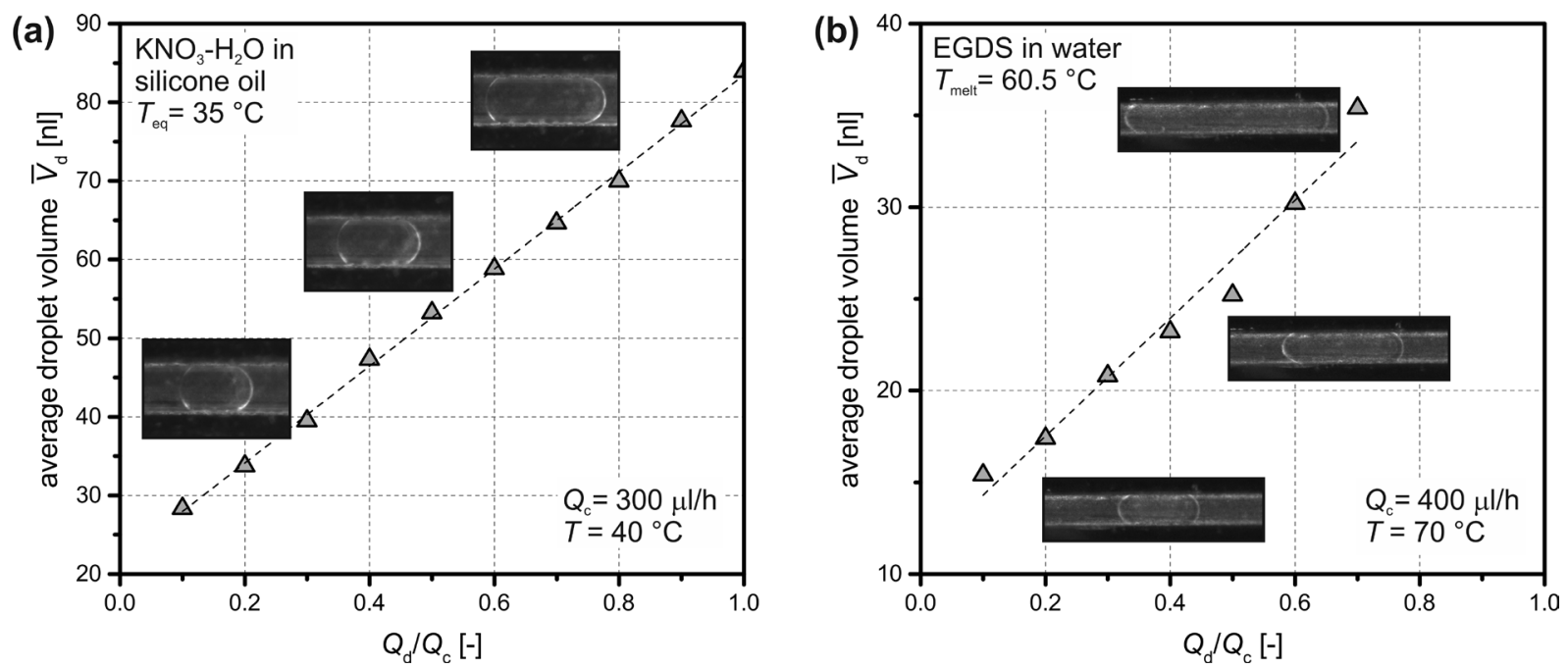

Figure 8. Evolution of the droplet volume as a function of the ratio of the dispersed phase to the continuous phase: (a) aqueous solution of $\mathrm{KNO}_{3}\left(T_{\mathrm{eq}}=35^{\circ} \mathrm{C}, X_{\mathrm{s}}=0.367 \mathrm{~kg}_{\mathrm{KNO} 3} / \mathrm{kg}_{\mathrm{sol}}\right)$ as dispersed phase and silicon oil as continuous phase at $40^{\circ} \mathrm{C}$, and (b) EGDS as dispersed phase and deionized water as continuous phase at $70^{\circ} \mathrm{C}$.

from the dimensions of a droplet or the channel geometry [36].

Figure 8 presents the evolution of the average droplet volume as a function of the flow rate ratio of the dispersed phase $Q_{\mathrm{d}}$ to the continuous phase $Q_{\mathrm{c}}$ for the aqueous solution of $\mathrm{KNO}_{3}$ (Figure 8(a)) and EGDS (Figure 8(b)). The flow rate of the continuous phase was kept constant at $300 \mu \mathrm{l} / \mathrm{h}$ for droplets of aqueous solution in silicone oil and at $Q_{\mathrm{c}}=400 \mu \mathrm{l} / \mathrm{h}$ for EGDS droplets in water. Each droplet volume was calculated from Equation (1) by counting at least 25 droplets. No significant change in the droplet volume determined was noticed with a higher amount of counted droplets. Both graphs show a steady increase in droplet volume with a higher ratio of volume flow rates of the dispersed phase to the continuous phase. Irregular droplet formation was observed for flow ratios above $Q_{\mathrm{d}} / Q_{\mathrm{c}}=0.7$ for EGDS. We measured the droplet length and the distances between two successive droplets while flowing through the channel with image analysis software (ImageJ) to check the monodispersity of the generated droplets. Small deviations of less than $5 \%$ were found.

\subsection{Crystallization Experiments}

We performed crystallization experiments for both aqueous $\mathrm{KNO}_{3}$ droplets in silicone oil and EGDS droplets in water to demonstrate the versatile usability of the microfluidic device for crystallization nucleation measurements. Up to 400 droplets were generated, as explained previously, with flow rate ratios of $\left(Q_{\mathrm{d}} / Q_{\mathrm{c}}\right)_{\mathrm{KNO3}}=0.4$ and $\left(Q_{\mathrm{d}} / Q_{\mathrm{c}}\right)_{\mathrm{EGDS}}=0.2$, and stored in the serpentine shaped part of the chip. Relatively small flow rate ratios were chosen to increase the number of droplets observable and increase the droplet distance to avoid interactions. Once a sufficient number of droplets was stored, the flow was stopped and the microfluidic device was cooled to the crystallization temperature $T_{\text {cryst }}$ desired. After approximately $60 \mathrm{~s}$, the final, isothermal temperature and, therefore, su- 
persaturation was achieved and kept constant during the remaining experiment.

First crystals can already be observed in the droplets during the cooling process at the conditions given. In the case of the aqueous $\mathrm{KNO}_{3}$ droplets, $\mathrm{KNO}_{3}$ crystals grow in the solution after nucleation until supersaturation is depleted. A detailed image of a $\mathrm{KNO}_{3}$ crystal is depicted in Figure 9(a). In contrast to $\mathrm{KNO}_{3}$, the whole EGDS droplet crystallizes after crystal nucleation occurred in the organic wax phase. This process only takes a few seconds (see Figure 9(b)).

The photographs in Figure 10 show a section of the storage area shortly after the beginning of the experiment and after approximately $1000 \mathrm{~s}$ and $2000 \mathrm{~s}$, respectively. Polarized light was used for better detection. Hence, crystals appear as bright pixels on the images recorded. Pictures were taken every second. Based on such pictures, the induction time of each individual crystallization event was recorded and the number of uncrystallized droplets was automatically counted by an image processing program as a function of time. Here, the induction time was taken as the time that elapses between the first buildup of supersaturation and the first occurrence of crystals. Together with the total number of droplets, the fraction of uncrystallized droplets $P(t)$ can be calculated to quantify crystallization.
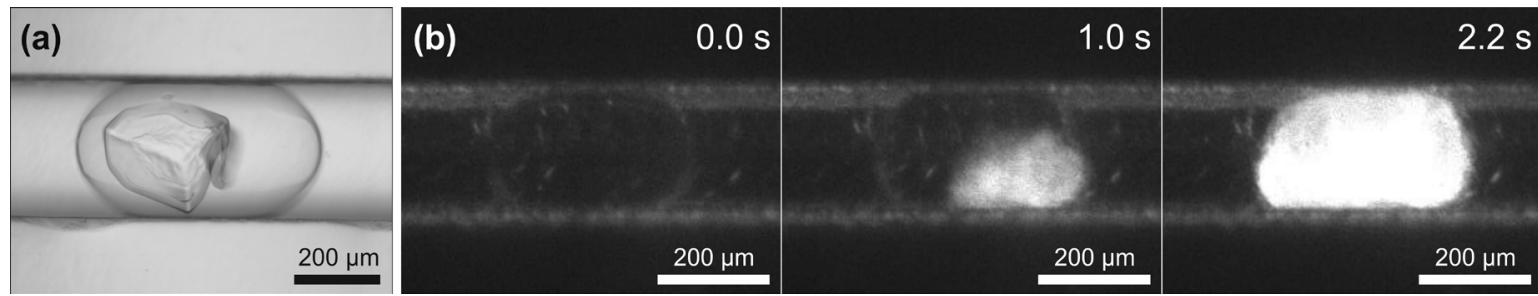

Figure 9. (a) Example of a $\mathrm{KNO}_{3}$ crystal in a solution droplet $\left(T_{\text {cryst }}=23^{\circ} \mathrm{C}\right)$; (b) crystallization of a EGDS droplet over time $\left(T_{\text {cryst }}=54.7^{\circ} \mathrm{C}\right)$. The whole droplet crystallizes within a few seconds.

(a)

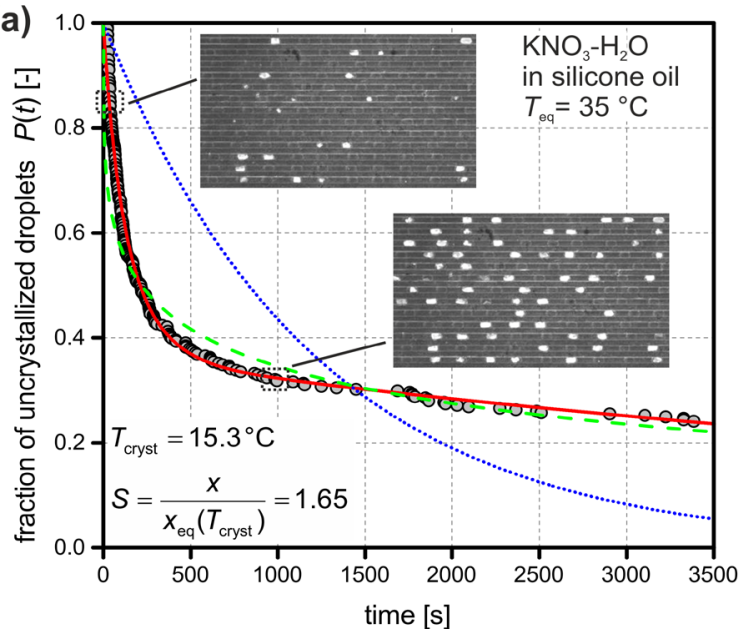

(b)

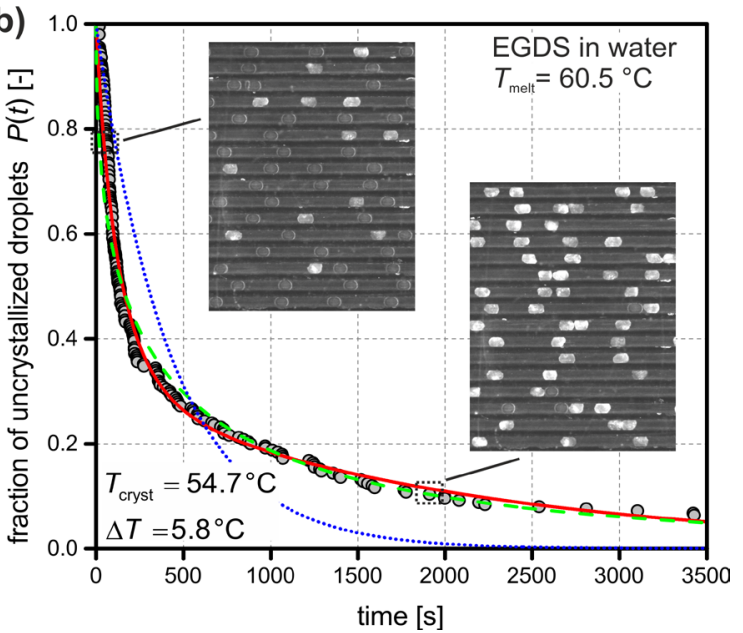

Figure 10. Temporal evolution of uncrystallized droplet fraction for (a) aqueous $\mathrm{KNO}_{3}$ droplets in silicone oil and (b) EGDS droplets in water. The photographs show a section of the storage area of the microfluidic device after different experimental times. The following models were applied to fit the experimental data: a single nucleation rate model (blue dotted line, Equation (2)), a Weibull model (green dashed line, Equation (3)) and the model of Pound and La Mer (red solid line, Equation (4)). 
As an example, the time evolution of this value is depicted in Figure 10 for aqueous $\mathrm{KNO}_{3}$ droplets in silicone oil at $T_{\text {cryst }}=15.3^{\circ} \mathrm{C}$ (Figure $10(\mathrm{a})$ ) and EGDS droplets in water at $T_{\text {cryst }}=54.7^{\circ} \mathrm{C}$ (Figure $10(\mathrm{~b})$ ). Solubility data for the solute solvent system $\mathrm{KNO}_{3} / \mathrm{H}_{2} \mathrm{O}$ was derived by using a correlation from [37]. A similar trend can be found for both substances at the experimental parameters given. With proceeding time, the fraction of uncrystallized droplets decreases. However, after a steep decay in the first $500 \mathrm{~s}$, the number of uncrystallized droplets decreases much more slowly at the end. We repeated the experiments several times. No significant change of the crystallization behavior was visible.

To describe and interpret the experimental data obtained, three different models from literature were used to fit the experimental data (see Figure 10): i) a model with a single nucleation rate [38], ii) an exponential of a power law (Weibull function) [39] and iii) the model of Pound and La Mer [40]. The corresponding equations are listed below.

i)

ii)

iii)

$$
\begin{gathered}
P(t)=\exp (-k \cdot t) \\
P(t)=\exp \left(-\left(t / \tau_{\text {med }}\right)^{\beta}\right) \\
P(t)=\exp [-m(1-\exp (k t))]+\exp (-m)\left[\exp \left(k_{0} t\right)-1\right]
\end{gathered}
$$

The first model assumes that the nucleation rate $k$ is constant over time $t$ and identical in all droplets, e.g. purely homogeneous or heterogeneous nucleation. In the Weibull model the nucleation rate is changing over time and is given by $k_{\mathrm{W}}(t)=\beta t^{(\beta-1)} / \tau_{\mathrm{med}}^{\beta} \cdot \beta$ is an exponent and the parameter $\tau_{\mathrm{med}}$ is related to the median nucleation time. In contrast, the model of Pound of La Mer assumes two different, constant nucleation rates, a heterogeneous nucleation rate $k$ and a homogeneous nucleation rate $k_{0}$. Heterogeneous nucleation centers are randomly distributed among the droplets. The average number of heterogeneous nucleation centers per droplet is given by $m$. Figure 10 shows the corresponding fitting curves. The model with a single nucleation rate (blue dotted line) fits the data insufficient. Both, the Weibull model (green dashed line) and the model of Pound and La Mer (red solid line) are capable to describe the experimental data well. Especially, considering that the Weibull model has just two parameters. So, none of these two models can be discarded and their explanations may be valid to explain droplet nucleation in our cases. The fit parameters for the models from Equations (3) and (4) are summarized in Table 1. Relating the fit parameters $\left(k, k_{0}\right)$ of Equation (4) to the droplet volume $\left(V_{\mathrm{d}, \mathrm{KNO} 3}=45.1 \mathrm{nl}\right.$ and $V_{\mathrm{d}, \mathrm{EGDS}}=$ $17.4 \mathrm{nl}$ ), volume specific nucleation rates are obtained. For $\mathrm{KNO}_{3}$ the volume specific heterogeneous nucleation rate is $11.5 \times 10^{7} \mathrm{~m}^{-3} \mathrm{~s}^{-1}$ and the volume specific homogeneous nucleation rate is $0.3 \times 10^{7} \mathrm{~m}^{-3} \mathrm{~s}^{-1}$. For EGDS we obtained values of $29.3 \times 10^{7} \mathrm{~m}^{-3} \mathrm{~s}^{-1}$ and $2.9 \times 10^{7} \mathrm{~m}^{-3} \mathrm{~s}^{-1}$.

It is noteworthy, that this behavior is present for both investigated substances, an inorganic salt solution as well as an organic melt. For this reason, it seems more reasonable that a general, non-substance-specific phenomenon, like active heterogeneous centers, is present. Therefore, we dedicate the first mechanism to 
Table 1. Fit parameters for the experimental data from Figure 10.

\begin{tabular}{cccc|cccc}
\hline & \multicolumn{6}{c}{ Model } \\
\cline { 2 - 8 } Substance & \multicolumn{3}{c}{ Weibull } & \multicolumn{4}{c}{ Pound and La Mer } \\
\cline { 2 - 8 } & \multicolumn{1}{c}{$\boldsymbol{C O D}\left(\mathrm{r}^{2}\right)$} & $\boldsymbol{\tau}$ & $\boldsymbol{\tau}_{\text {med }}[\mathbf{s}]$ & $\mathrm{COD}\left(\mathrm{r}^{2}\right)$ & $\boldsymbol{k}\left[10^{-3} \mathbf{s}^{-1}\right]$ & $\boldsymbol{k}_{0}\left[10^{-5} \mathbf{s}^{-1}\right]$ & $\boldsymbol{m}$ \\
\hline $\mathrm{KNO}_{3} / \mathrm{H}_{2} \mathrm{O}$ & 0.89 & $0.28 \pm 0.01$ & $800 \pm 6$ & 0.99 & $5.2 \pm 0.02$ & $12.2 \pm 0.06$ & $1.01 \pm 0.01$ \\
EGDS & 0.96 & $0.47 \pm 0.02$ & $331 \pm 1$ & 0.99 & $5.1 \pm 0.04$ & $50.1 \pm 0.35$ & $1.20 \pm 0.01$ \\
\hline
\end{tabular}

heterogeneous nucleation centers which are randomly distributed inside of the droplets. Following the work of Pound and La Mer [40], the second slope could be attributed to homogeneous nucleation. However, other microfluidic studies showed that some kind of heterogeneous nucleation is more likely to be the underlying nucleation mechanism [9] [11]. Further investigation is necessary to gain evidence of the active nucleation mechanisms and is currently in progress.

\section{Conclusions}

In this paper, the suitability of a simple generic microfluidic tool for investigating crystallization is demonstrated. As microfluidic devices to conduct crystallization experiments are mainly made of PDMS, this polycarbonate based tool can be seen as an alternative. Since PC is a material with good machining properties, we proofed the fabrication of well-defined microfluidic devices with a high flexibility in chip geometry by using a milling machine. Examination of the channel surfaces with electron microscopy and a noninvasive optical method showed that the surface roughness is in the same magnitude as materials used in common crystallization apparatuses.

Another benefit of PC is that reliable surface modification can be achieved easily. As the successful droplet generation depends strongly on the surface wetting properties of the microfluidic device, this allows for the generation of droplets of both substances which require hydrophobic or hydrophilic surface properties. In our work, we demonstrated the controlled segmentation of aqueous $\mathrm{KNO}_{3}$ solution and EGDS in highly monodisperse droplets using a T-junction. No significant change in droplet generation and volume was noticed over several sets of experiments using the same microfluidic device.

The suitability of this setup to investigate crystal nucleation for various applications was shown by means of cooling crystallization experiments on aqueous $\mathrm{KNO}_{3}$ solution and EGDS. To this end, the induction time of each individual crystallization event during the experiments was determined using image analysis software. From this data, we derived the evolution of the fraction of uncrystallized droplets over time. Thus, using this experimental setup, the influence of different conditions, for example, temperature, on crystal nucleation can be examined. Further, it is possible to derive nucleation kinetics and examine underlying mechanisms.

With respect to our results, we believe that our approach extends the common microfluidic methods and enables new opportunities to investigate crystallization. 


\section{Acknowledgements}

Financial support for this work by the AIF-Forschungsvereinigung (ForschungsGesellschaft Verfahrens-Technik e.V, Project no. $18462 \mathrm{~N}$ ) is gratefully acknowledged. The authors also thank Andreas Roth from the workshop for his active support during the milling procedure of the microfluidic devices. Furthermore, the authors would like to thank their colleagues from the Institute for Applied Materials (IAM-WK) at KIT for their support with the electron microscopy pictures.

\section{References}

[1] Abramov, S., Ruppik, P. and Schuchmann, H. (2016) Crystallization in Emulsions: A Thermo-Optical Method to Determine Single Crystallization Events in Droplet Clusters. Processes, 4, 25. https://doi.org/10.3390/pr4030025

[2] McClements, D.J. (2012) Crystals and Crystallization in Oil-In-Water Emulsions: Implications for Emulsion-Based Delivery Systems. Advances in Colloid and Interface Science, 174, 1-30. https://doi.org/10.1016/j.cis.2012.03.002

[3] Gebauer, J. and Kind, M. (2015) Experimental Screening Method for FlashCrystallization. Chemical Engineering Science, 133, 75-81. https://doi.org/10.1016/j.ces.2014.12.034

[4] Shi, H.-H., Xiao, Y., Ferguson, S., Huang, X., Wang, N. and Hao, H.-X. (2017) Progress of Crystallization in Microfluidic Devices. Lab on a Chip, 17, 2167-2185. https://doi.org/10.1039/C6LC01225F

[5] Chen, D.L., Gerdts, C.J. and Ismagilov, R.F. (2005) Using Microfluidics to Observe the Effect of Mixing on Nucleation of Protein Crystals. Journal of the American Chemical Society, 127, 9672-9673. https://doi.org/10.1021/ja052279v

[6] Shim, J.-U., Cristobal, G., Link, D.R., Thorsen, T. and Fraden, S. (2007) Using Microfluidics to Decouple Nucleation and Growth of Protein Crystals. Crystal Growth \& Design, 7, 2192-2194. https://doi.org/10.1021/cg700688f

[7] Maeki, M., Teshima, Y., Yoshizuka, S., Yamaguchi, H., Yamashita, K. and Miyazaki, M. (2014) Controlling Protein Crystal Nucleation by Droplet-Based Microfluidics. Chemistry, 20, 1049-1056. https://doi.org/10.1002/chem.201303270

[8] Pham, N., Radajewski, D., Round, A., Brennich, M., Pernot, P., Biscans, B., Bonnete, F. and Teychene, S. (2017) Coupling High Throughput Microfluidics and Small-Angle X-Ray Scattering to Study Protein Crystallization from Solution. Analytical Chemistry, 89, 2282-2287. https://doi.org/10.1021/acs.analchem.6b03492

[9] Teychené, S. and Biscans, B. (2011) Microfluidic Device for the Crystallization of Organic Molecules in Organic Solvents. Crystal Growth \& Design, 11, 4810-4818. https://doi.org/10.1021/cg2004535

[10] Maeki, M., Yamaguchi, H., Tokeshi, M. and Miyazaki, M. (2016) Microfluidic Approaches for Protein Crystal Structure Analysis. Analytical Sciences the International Journal of the Japan Society for Analytical Chemistry, 32, 3-9. https://doi.org/10.2116/analsci.32.3

[11] Teychené, S. and Biscans, B. (2012) Crystal Nucleation in a Droplet Based Microfluidic Crystallizer. Chemical Engineering Science, 77, 242-248.

https://doi.org/10.1016/j.ces.2012.01.036

[12] Gerdts, C.J., Elliott, M., Lovell, S., Mixon, M.B., Napuli, A.J., Staker, B.L., Nollert, P. and Stewart, L. (2008) The Plug-Based Nanovolume Microcapillary Protein Crystal- 
lization System (MPCS). Acta Crystallographica. Section D, Biological Crystallography, 64, 1116-1122. https://doi.org/10.1107/S0907444908028060

[13] Dombrowski, R.D., Litster, J.D., Wagner, N.J. and He, Y. (2007) Crystallization of Alpha-Lactose Monohydrate in a Drop-Based Microfluidic Crystallizer. Chemical Engineering Science, 62, 4802-4810. https://doi.org/10.1016/j.ces.2007.05.033

[14] Laval, P., Salmon, J.-B. and Joanicot, M. (2007) A Microfluidic Device for Investigating Crystal Nucleation Kinetics. Journal of Crystal Growth, 303, 622-628. https://doi.org/10.1016/j.jcrysgro.2006.12.044

[15] Lu, J., Litster, J.D. and Nagy, Z.K. (2015) Nucleation Studies of Active Pharmaceutical Ingredients in an Air-Segmented Microfluidic Drop-Based Crystallizer. Crystal Growth \& Design, 15, 3645-3651. https://doi.org/10.1021/acs.cgd.5b00150

[16] Vitry, Y., Teychené, S., Charton, S., Lamadie, F. and Biscans, B. (2015) Investigation of a Microfluidic Approach to Study Very High Nucleation Rates Involved in Precipitation Processes. Chemical Engineering Science, 133, 54-61. https://doi.org/10.1016/j.ces.2015.01.062

[17] Kashchiev, D. (2000) Nucleation: Basic Theory with Applications. Butterworth Heinemann, Oxford, Boston.

[18] Brandel, C., Ter, H. and Joop, H. (2015) Measuring Induction Times and Crystal Nucleation Rates. Faraday Discussions, 179, 199-214. https://doi.org/10.1039/C4FD00230J

[19] Jiang, S., Ter, H. and Joop, H. (2011) Crystal Nucleation Rates from Probability Distributions of Induction Times. Crystal Growth \& Design, 11, 256-261. https://doi.org/10.1021/cg101213q

[20] Jankowski, P., Ogonczyk, D., Kosinski, A., Lisowski, W. and Garstecki, P. (2011) Hydrophobic Modification of Polycarbonate for Reproducible and Stable Formation of Biocompatible Microparticles. Lab on a Chip, 11, 748-752. https://doi.org/10.1039/C0LC00360C

[21] Bico, J. and Quere, D. (2002) Self-Propelling Slugs. Journal of Fluid Mechanics, 467, 101-127. https://doi.org/10.1017/S002211200200126X

[22] Bico, J. and Quere, D. (2002) Rise of Liquids and Bubbles in Angular Capillary Tubes. Journal of Colloid and Interface Science, 247, 162-166. https://doi.org/10.1006/jcis.2001.8106

[23] Whitesides, G.M. (2006) The Origins and the Future of Microfluidics. Nature, 442, 368-373. https://doi.org/10.1038/nature05058

[24] Becker, H. (2002) Polymer Microfluidic Devices. Talanta, 56, 267-287. https://doi.org/10.1016/S0039-9140(01)00594-X

[25] Sia, S.K. and Whitesides, G.M. (2003) Microfluidic Devices Fabricated in Poly (Dimethylsiloxane) for Biological Studies. Electrophoresis, 24, 3563-3576. https://doi.org/10.1002/elps.200305584

[26] McDonald, J.C. and Whitesides, G.M. (2002) Poly(dimethylsiloxane) as a Material for Fabricating Microfluidic Devices. Accounts of Chemical Research, 35, 491-499. https://doi.org/10.1021/ar010110q

[27] Lo, R. (2013) Application of Microfluidics in Chemical Engineering. Journal of Chemical Engineering and Process Technology, 1002-1005.

[28] Lee, J.N., Park, C. and Whitesides, G.M. (2003) Solvent Compatibility of Poly (dimethylsiloxane)-Based Microfluidic Devices. Analytical Chemistry, 75, 6544-6554. https://doi.org/10.1021/ac0346712

[29] Dangla, R., Gallaire, F. and Baroud, C.N. (2010) Microchannel Deformations Due to 
Solvent-Induced PDMS Swelling. Lab on a Chip, 10, 2972-2978. https://doi.org/10.1039/c003504a

[30] Wang, Y., He, Q., Dong, Y. and Chen, H. (2010) In-Channel Modification of Biosensor Electrodes Integrated on a Polycarbonate Microfluidic Chip for Micro Flow-Injection Amperometric Determination of Glucose. Sensors and Actuators B: Chemical, 145, 553-560. https://doi.org/10.1016/j.snb.2009.11.068

[31] Witek, M.A., Hupert, M.L., Park, D.S.-W., Fears, K., Murphy, M.C. and Soper, S.A. (2008) 96-Well Polycarbonate-Based Microfluidic Titer Plate for High-Throughput Purification of DNA and RNA. Analytical Chemistry, 80, 3483-3491. https://doi.org/10.1021/ac8002352

[32] Ogonczyk, D., Wegrzyn, J., Jankowski, P., Dabrowski, B. and Garstecki, P. (2010) Bonding of Microfluidic Devices Fabricated in Polycarbonate. Lab on a Chip, 10, 1324-1327. https://doi.org/10.1039/b924439e

[33] Qi, H., Chen, T., Yao, L. and Zuo, T. (2009) Micromachining of Microchannel on the Polycarbonate Substrate with $\mathrm{CO}_{2}$ Laser Direct-Writing Ablation. Optics and Lasers in Engineering, 47, 594-598. https://doi.org/10.1016/j.optlaseng.2008.09.004

[34] Derzsi, L., Jankowski, P., Lisowski, W. and Garstecki, P. (2011) Hydrophilic Polycarbonate for Generation of Oil in Water Emulsions in Microfluidic Devices. Lab on a Chip, 11, 1151-1156. https://doi.org/10.1039/c0lc00438c

[35] Lee, E.-S. (2000) Machining Characteristics of the Electropolishing of Stainless Steel (STS316L). The International Journal of Advanced Manufacturing Technology, 16, 591-599. https://doi.org/10.1007/s001700070049

[36] Musterd, M., van Steijn, V., Kleijn, C.R. and Kreutzer, M.T. (2015) Calculating the Volume of Elongated Bubbles and Droplets in Microchannels from a Top View Image. RSC Advances, 5, 16042-16049. https://doi.org/10.1039/C4RA15163A

[37] Kaiser, R. (2009) Flash-Kristallisation. Ein neues Verfahren zur Produktgestaltung kristalliner Güter. PhD Dissertation, Technische Universität Karlsruhe, Karlsruhe.

[38] Turnbull, D. (1952) Kinetics of Solidification of Supercooled Liquid Mercury Droplets. Journal of Chemical Physics, 20, 411. https://doi.org/10.1063/1.1700435

[39] Sear, R.P. (2014) Quantitative Studies of Crystal Nucleation at Constant Supersaturation: Experimental Data and Models. CrystEngComm, 16, 6506-6522. https://doi.org/10.1039/C4CE00344F

[40] Pound, G.M. and La Mer, V.K. (1952) Kinetics of Crystalline Nucleus Formation in Supercooled Liquid Tin. Journal of the American Chemical Society, 74, 2323-2332. https://doi.org/10.1021/ja01129a044 